\title{
EXPERIMENT AND NUMERICAL SIMULATION OF REPEATED LIQUEFACTION-CONSOLIDATION OF SAND
}

\author{
Bin Ye ${ }^{\mathrm{i}}$, Guanlin Ye ${ }^{\mathrm{ii})}$, Feng Zhang ${ }^{\mathrm{iii}}$ and Atsushi Yashima ${ }^{\mathrm{iv})}$
}

\begin{abstract}
Behaviors of a liquefied ground are influenced by many factors, such as density, confining stress, permeability, particle size and grading, etc. Among these factors, the density plays the most significant role. It is known that when subjected to cyclic loading under undrained condition, there exist three types of liquefaction behaviors, i.e., loose sand will fail directly towards zero mean effective stress without cyclic mobility; medium-dense sand will enter into a cyclic mobility before being completely liquefied; very dense sand, however, will not liquefy at all. In liquefaction analysis, it is important to distinguish these three types of liquefaction behaviors according to different densities. In this paper, firstly, a constitutive model proposed by the same authors is briefly introduced. Secondly, experimental results of shaking-table tests on saturated sandy ground with repeated liquefaction-consolidation process are presented. Then, a finite element-finite difference method (FE-FD) based on the constitutive model and two-phase field theory is conducted. Comparisons between the experiment and the numerical simulation show that the numerical simulation is capable of reproducing almost all main characteristics of the repeated liquefaction-consolidation of sandy grounds with different densities, such as the mechanical behavior pre- and during liquefaction, the settlement in post-liquefaction consolidation and the influence of density on the accumulation of excessive pore water pressure (EPWP) in repeated strong motions.
\end{abstract}

Key words: consolidation, cyclic mobility, liquefaction, numerical analysis, shaking table test (IGC: D6)

\section{INTRODUCTION}

Liquefaction is one of the most dangerous threats to structures constructed in a sandy ground during an earthquake. Researchers have made great efforts to make clear the mechanism of liquefaction in sandy soil experimentally and numerically. The behaviors of a liquefied ground are influenced by many factors, such as density, confining stress, permeability, particle size and grading, etc. Among these factors, density plays the most significant role. It is well known that when subjected to cyclic shear loading under undrained condition, there exist three types of liquefaction behaviors, i.e., loose sand (No. 1 silica sand, relative density $=0.1$, Nakai, 2005) will fail directly towards zero mean effective stress without cyclic mobility, as shown in Fig. 1(a); mediumdense sand (No. 1 silica sand, relative density $=0.58$, Nakai, 2005) will enter into a cyclic mobility before being completely liquefied, as shown in Fig. 1(b); very dense sand, however, will not liquefy at all. In liquefaction analysis, it is important to distinguish these three types of liquefaction behaviors according to different densities.

On the other hand, liquefaction analysis still remains a
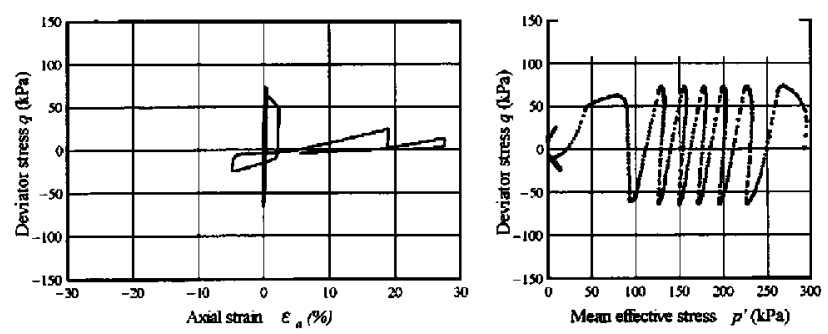

(a) loose sand (fail directly without cyclic mobility)
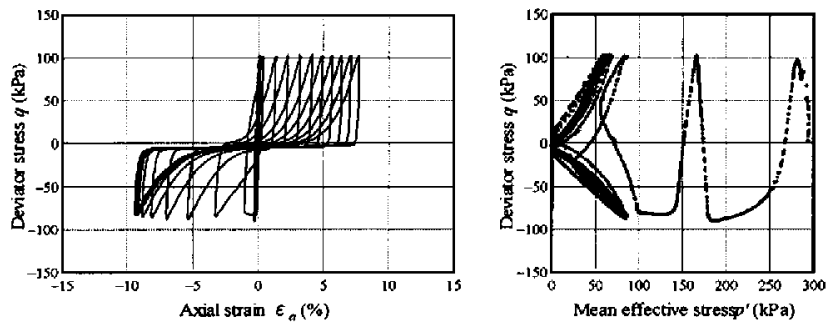

(b) medium dense sand (cyclic mobility)

Fig. 1. Mechanical behaviors of loose sand and medium dense sand subjected to cyclic triaxial shear tests under undrained condition (Nakai, 2005)

i) Graduate Student, Department of Civil Engineering, Gifu University, Japan.

ii) Researcher, Geo-Research Institute, Japan.

iii) Professor, Department of Civil Engineering, Nagoya Institute of Technology, Japan (zhang-f@tntil.ace.nitech.ac.jp).

iv) Professor, Department of Civil Engineering, Gifu University, Japan.

The manuscript for this paper was received for review on August 28, 2006; approved on January $15,2007$.

Written discussions on this paper should be submitted before January 1, 2008 to the Japanese Geotechnical Society, 4-38-2, Sengoku, Bunkyo-ku, Tokyo 112-0011, Japan. Upon request the closing date may be extended one month. 
challenge to researchers. This is because that liquefaction analysis involves three difficult but key points: 1) a constitutive model that can properly describe cyclic behaviors of saturated sands with different densities; 2) a soil-water coupling two-phase theory that can take into account the interaction between soil and pore-water; 3 ) a numerical method that can give a stable and accurate solution of a boundary value problem (BVP) especially when effective stress of soils is extremely small during liquefaction.

As to the first point, researchers have proposed a lot of cyclic constitutive models for sand, e.g., Pastor and Zienkiewicz (1986); Nishi and Kanatani (1990); Ishihara and Kabilamany (1990); Oka et al. (1992, 1999); Elgamal et al. (2003); Zhang and Wang (2005). All these models can describe the cyclic behaviors of saturated sands under cyclic loading to some extent, but they have to prescribe different values to material parameters for sands with different densities in order to distinguish the three types of liquefaction behaviors, even if the same sand is dealt with. As we know, however, the density of sand will change greatly in a liquefaction process of BVP because pore water dissipates from ground. So it is obviously not reasonable to use different values of material parameters for soils with different densities. Zhang et al. (2006) proposed a new constitutive model that can describe the different cyclic behaviors of sands with different densities using the same values of material parameters, i.e., the values of material parameters do not depend on density but just depend on what kind of sands it deals with.

As to the second and third points, Oka et al. (1991, 1994) proposed a soil-water coupling two-phase field theory with u-p formulation. Combing this strict twophase theory and a cyclic constitutive model, it is possible to solve a BVP of liquefaction in soils by a soil-water coupling finite element-finite difference analysis (Yashima et al., 1991).

In this paper, firstly, the newly proposed model (Zhang et al., 2006) is briefly introduced. Secondly, experimental results of shaking-table tests on saturated sandy ground with repeated liquefaction-consolidation process are presented. Then, a finite element-finite difference method (FE-FD) based on the newly proposed and the two-phase field theory proposed by Oka et al. $(1991,1994)$ is conducted. Comparisons between the experiment and the numerical simulation show that the numerical simulation is capable of reproducing almost all main characteristics of the repeated liquefaction-consolidation of sandy grounds with different densities, such as the mechanical behavior pre- and during liquefaction, the settlement in post-liquefaction consolidation and the influence of density on the accumulation of excessive pore water pressure (EPWP) in repeated strong motions.

\section{A NEW CONSTITUTIVE MODEL FOR SOILS}

The newly proposed model (Zhang et al., 2006) is an elasto-plastic model, which adopted some important concepts such as stress-induced anisotropy (Sekiguchi,
1977), subloading yield surface (Hashiguchi and Ueno, 1977; Hashiguchi, 1978, 1989), and superloading yield surface (Asaoka et al., 1998, 2000a, 2000b, 2002). The model has some distinct characteristics as follows:

a) A new type of yield surface with fixed critical state line in stress space is proposed;

b) A new type of stress-induced anisotropy and its correspondent evolution are proposed;

c) A new type of evolution rule for overconsolidation, taking into consideration of the stress-induced anisotropy, is proposed;

d) An associated flow rule is adopted so that the formulation of the model is very simple;

e) It is able to describe different behaviors of both sand and clay;

f) It is able to describe the different cyclic behaviors of sands with different densities using the same values of material parameters;

g) It is able to describe mechanical behaviors of soils under both monotonic loading and cyclic loading.

Eight parameters are involved in the proposed model, among which five parameters, $\mathrm{M}, \mathrm{N}, \tilde{\lambda}, \tilde{\kappa}$, and $v$ are the same as in the Cam-clay model. The other three parameters are listed below.

$a$ : parameter which controls the collapse rate of structure when the soil is subjected to shearing or compression. It can be determined by triaxial compression tests on remold soil and undisturbed soil, considering the difference of peak strengths between the soils.

$m$ : parameter which controls the losing rate of overconsolidation when the soil subjected to shearing or compression. It can be determined by triaxial compression tests on normally-consolidated soil and overconsolidated soil, considering the difference of peak strengths between the soils.

$b_{\mathrm{r}}$ : parameter which controls the developing rate of stress-induced anisotropy when the soil subjected to shearing or compression. It can be determined based on the performance of the soil which is influenced by the development of the stress-induced anisotropy.

The details of this model can be referred to the references (Zhang et al., 2006). Figure 2 shows element simulations of loose sand, medium-dense sand and dense sand under cyclic loading with undrained condition. The sand considered here is Toyoura Standard Sand. The purpose of the figure is just to show the performance of the model. Material parameters and initial state parameters are shown in Tables 1 and 2. It is very clear from the figure that loose sand will fail directly towards zero effective stress state without the process of cyclic mobility; for medium-dense sands, liquefaction will occur with the cyclic mobility, dense sand, however, will never liquefy. This performance is coincident with the test results that can be found in many literatures, for instance, the results shown in Fig. 1. Of course, the model is not always perfect. As can be seen in Figs. 1 and 2, for medium-dense sands, after the cyclic mobility, shear 

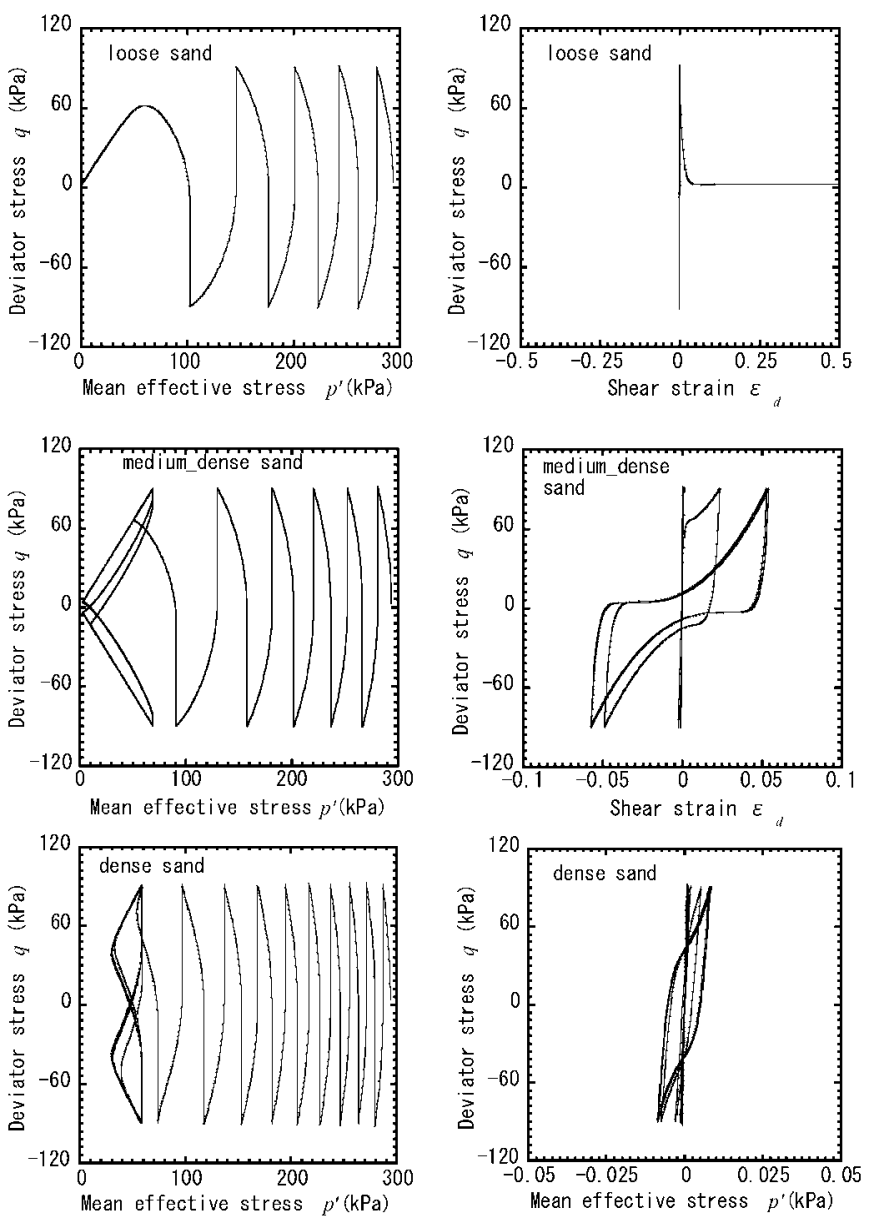

Fig. 2. Element simulation of sands with different densities $\left(p_{0}^{\prime}=\mathbf{2 9 8}\right.$ $\mathrm{kPa})$

Table 1. Material parameters of Toyoura Sand

\begin{tabular}{ll}
\hline Compression index $\lambda$ & 0.05 \\
Swelling index $\boldsymbol{\kappa}$ & 0.0064 \\
Critical state parameter M & 1.30 \\
Void ratio N $\left(p^{\prime}=98 \mathrm{kPa}\right.$ on N.C.L.) & 0.74 \\
Poisson's ratio $\boldsymbol{v}$ & 0.30 \\
\hline Degradation parameter of overconsolidation state $\boldsymbol{m}$ & 0.10 \\
Degradation parameter of structure $\boldsymbol{a}$ & 2.2 \\
Evolution parameter of anisotropy $\boldsymbol{b}_{\mathrm{r}}$ & 1.5 \\
\hline
\end{tabular}

Table 2. Initial conditions of sands with different densities

\begin{tabular}{lccc}
\hline & $\begin{array}{c}\text { Loose } \\
\text { sand }\end{array}$ & $\begin{array}{c}\text { Medium- } \\
\text { dense sand }\end{array}$ & $\begin{array}{c}\text { Dense } \\
\text { sand }\end{array}$ \\
\hline Initial void ratio $\boldsymbol{e}_{0}$ & $0.81\left(D_{\mathrm{r}}=0.44\right)$ & $0.70\left(D_{\mathrm{r}}=0.75\right)$ & $0.63\left(D_{\mathrm{r}}=0.92\right)$ \\
\hline Initial mean effective stress $\boldsymbol{p}^{\prime}(\mathrm{kPa})$ & 294 & 294 & 294 \\
\hline Initial degree of structure $\boldsymbol{R}_{0}^{*}$ & 0.0095 & 0.40 & 0.70 \\
\hline Initial degree of overconsolidation $1 / \boldsymbol{R}_{0}$ & 1.19 & 2.0 & 5.07 \\
\hline Initial anisotropy $\zeta_{0}$ & 0.0 & 0.0 & 0.00 \\
\hline
\end{tabular}

strain increases gradually in test while the simulation sometimes shows a rather abrupt change, though the model can simulate the gradual increase of shear strain in cyclic mobility region (Zhang et al., 2006). It should be noticed that the values of material parameters for three sand samples are the same. These three sands with different densities are just prepared by vibration compaction from the same state with different numbers of compactions, and the amplitude of the vibration is $2.3 \mathrm{kPa}$. After the compaction, these sands with different densities are then isotropically consolidated to a prescribed confining pressure of $294 \mathrm{kPa}$. Detailed description of the preparation of these sands can be referred to at the reference (Zhang et al., 2006).

\section{SHAKING-TABLE TESTS AND NUMERICAL SIMULATION}

\section{Brief Description of Shaking-table Tests}

In order to investigate the different behaviors of sands with different densities, a series of $1 \mathrm{G}$ shaking-table tests on saturated sandy grounds with different densities were conducted in Gifu University (Ye et al., 2006). The model ground is shown in Fig. 3. The model ground was made of Toyoura Sand, which is widely used in geotechnical experiments in Japan. Accelerations and EPWP were measured at different depths of the model ground.

An initial loose sandy ground, with void ratio of about 0.78 and relative density of $53 \%$, was prepared carefully by 'water sedimentation method', in which, firstly, water with a depth of $10 \mathrm{~cm}$ was poured into the shear box, and then saturated sand was poured into the shear box slowly and carefully by scoop beneath water level. The model ground was built up little by little until the ground reached the height of about $60 \mathrm{~cm}$.

The prepared ground was shaken three times in succession, marked by Case 1, Case 2 and Case 3, and the corresponding physical quantities are listed in Table 3. The shakings in Case 2 and Case 3 were applied to the model ground after the EPWP built up in the previous case had dissipated completely. The model ground became denser and denser after the repeated vibrations due to the dissipation of pore water in the model ground during and after each shaking. The input waves in all

Note: $e_{\max }=0.97 ; e_{\min }=0.61$ 


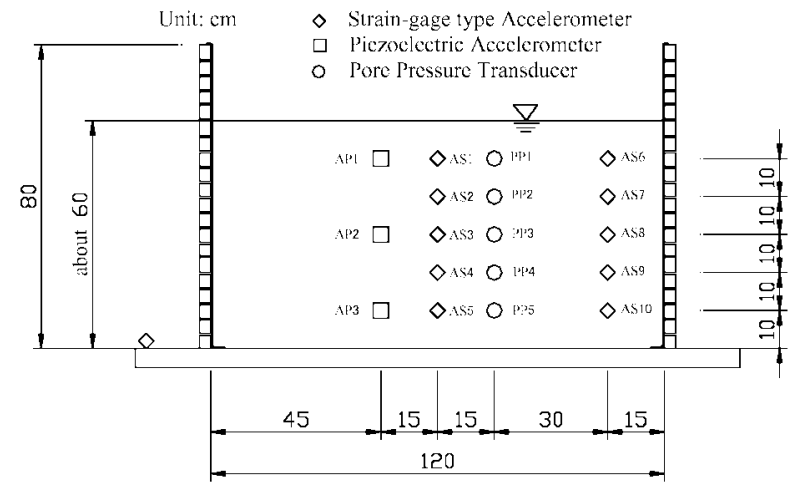

Fig. 3. The model ground of shaking-table tests (Ye et al., 2006)

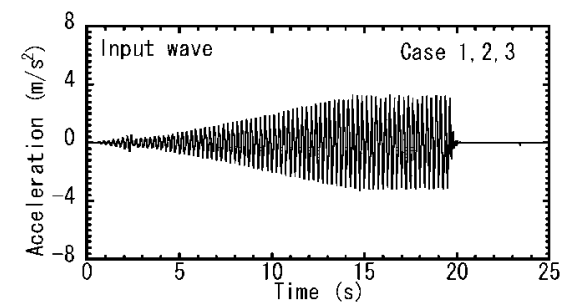

Fig. 4. The input wave

Table 3. Cases of shaking-table tests

\begin{tabular}{lcccc}
\hline Case & $\begin{array}{c}\text { Average void } \\
\text { ratio before } \\
\text { shaking }\end{array}$ & $\begin{array}{c}\text { Relative } \\
\text { density }\end{array}$ & $\begin{array}{c}\text { Input } \\
\text { wave }\end{array}$ & Frequency \\
\cline { 1 - 3 } 1 & 0.78 & $52 \%$ & & \\
\cline { 1 - 3 } 2 & 0.75 & $61 \%$ & sine & $4 \mathrm{~Hz}$ \\
\cline { 1 - 3 } 3 & 0.70 & $74 \%$ & & \\
\hline
\end{tabular}

cases are the same, as shown in Fig. 4. It is a sweep wave increased from zero to $300 \mathrm{Gal}$.

\section{Numerical Methods and Simulation Procedures}

The newly proposed model has been implemented into a program called DBLIVE. This $2 \mathrm{D}$ and $3 \mathrm{D}$ FEM program is capable of solving repeated static and dynamic soil-water coupled BVP by using Oka's two-phase field theory (Oka et al., 1991, 1994) and FE-FD soil-water coupled method. In this subsection, the shaking-table tests described in the previous subsection was simulated using the program DBLIVE.

Because the model ground is a level ground, the FEM model can be simplified to a one-dimensional column of soil elements, as shown in Fig. 5. Here we assume that the ground is uniform across any lateral plane.

The boundary condition is that:

(a) The bottom of the ground is fixed;

(b) In dynamic analysis, an equal-displacement-boundary condition (Yashima et al., 1991) is used between the nodes in two sides of the ground; when the analysis is shifted to consolidation, the boundary condition is then changed to fixed one.

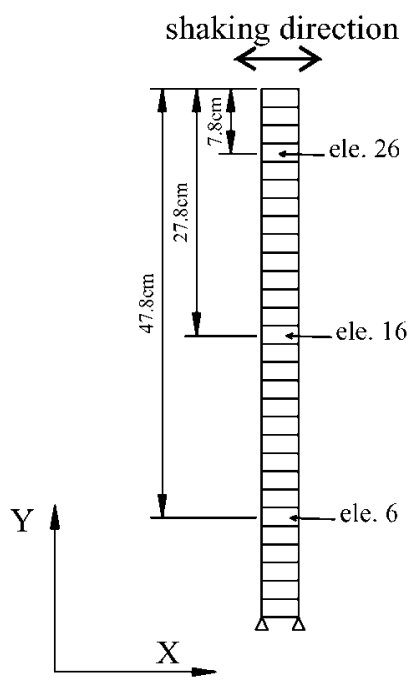

Fig. 5. The simplified FEM mesh

Table 4. Initial conditions of sands in numerical simulation

$\begin{array}{lc}\text { Initial void ratio } \boldsymbol{e}_{0} & 0.78 \\ \text { Initial mean effective stress } p^{\prime}(\mathrm{kPa}) & 2.50 \\ \text { Initial degree of structure } \boldsymbol{R}_{0}^{*} & 0.80 \\ \text { Initial degree of overconsolidation } 1 / \boldsymbol{R}_{0} & 25.0 \\ \text { Initial anisotropy } \zeta_{0} & 0.00\end{array}$

(c) Ground surface is set as drainage condition while the other surfaces are impermeable.

The input motion is the same as what was used in the experiment, as shown in Fig. 4. The numerical simulation aims to reproduce the three cases of shaking-table tests, in which the model ground was shaken three times in succession and became denser and denser after each shaking. In the numerical calculation, the process during shaking is simulated by a dynamic soil-water coupled analysis, while the process of dissipation of EPWP is simulated by a static consolidation analysis. The whole process of the model tests, which consists of three shakings and three consolidations, is a continuous one and therefore it should be simulated in sequential continuously. The simulation is therefore divided into six stages, i.e., three stages of dynamic analysis and three stages of consolidation analysis. The dynamic analysis lasts for 20 seconds until shaking stops, and the consolidation analysis lasts for 1000 seconds until EPWP dissipates completely. The values of material parameters, listed in Table 1, are the same in all 6 stages. Five initial state parameters before shaking are listed in Table 4. The state of soil elements valuated by these five state parameters is only prescribed at the very beginning and the values of these parameters will be delivered automatically to the next stage of the analysis in the whole process, which is totally the same as what been done in the model test. The initial stress filed is calculated by a self-weight static analysis in advance.

The element simulation of the initial soil element is shown in Fig. 6. It is worth noticing that the confining 
pressure of the ground in the shaking-table test is very small if compared to those used in conventional triaxial compression tests due to a very shallow depth of $0.6 \mathrm{~m}$ for the model ground. Therefore, a rather large value of the initial OCR in Table 4 is used.

\section{Comparison of the Simulation and the Experiment}

Figure 7 shows the test results of acceleration responses at different depths in different cases. In Case 1, it can be seen that after liquefaction the acceleration responses became very small throughout the ground, implying that the loose ground liquefied completely. Strictly speaking, liquefaction means the situation in which, the effective stress reduce to zero and the EPWP keeps the value of the initial effect stress during shaking. This can be verified with the time change of EPWP. It can also be predicted indirectly with the time change of the responding acceleration of surface ground because after liquefaction the responding acceleration of the ground above the liquefied soil layer became very small. In Case 2 and Case 3 , the acceleration responses remained small in the ground near surface but keep large in deeper places, showing that after experiencing liquefaction, the densities
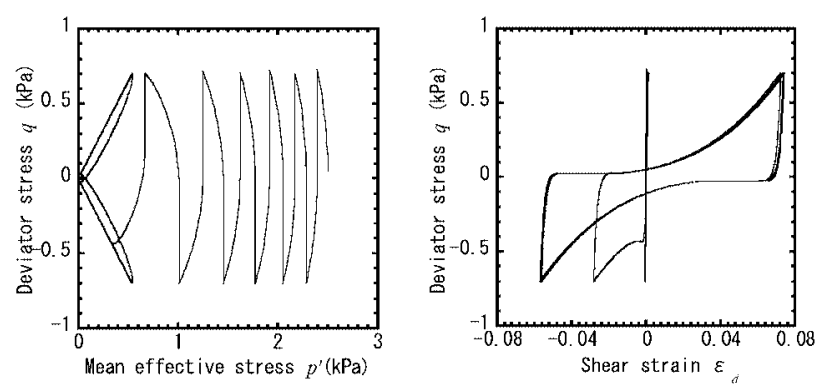

Fig. 6. Element simulation at low confining pressure $\left(p_{0}^{\prime}=2.5 \mathrm{kPa}\right)$ of sands increased and the sands in deeper places may not liquefy in the next vibration.

Figure 8 shows the simulated results of acceleration responses at different depths in different cases. It can be seen that acceleration responses of ground increased with ground becoming denser and denser case by case, which shows the same tendency as the experimental results. In every case, acceleration was small near ground surface, and increased along with the increase of depth. Experimental results also show this trend in Case 2 and Case 3. However, in Case 1, acceleration of experiment became very small throughout the ground after liquefaction, while simulated result still gave a rather large value of acceleration in deep place. This disagreement is mainly caused by the method we used to calculate the damping matrix. Damping is difficult to be determined for liquefaction analysis. In this simulation we used stiffness proportional damping in dynamic analysis, i.e., damping matrix $[C]$ is calculated by the relation $[C]=\alpha[K]$, where $\alpha$ is the proportional constant and $[K]$ is the stiffness matrix. In order to obtain a stable numerical solution, here we let damping matrix be proportional to the initial stiffness matrix $\left[K_{0}\right]$. As a result, the calculated acceleration response will not be decreased to zero even if the ground is fully liquefied because a relatively large value of damping remained after full liquefaction. Another reason might be the inconsistency existed in the stressstrain relation predicted by the model as mentioned in the comment for Figs. 1 and 2, that is, the gradual increase of shear strain in cyclic mobility region sometime can not be perfectly simulated by the model yet.

Figure 9 shows the EPWP measured at different depths in different cases. It is noticeable that EPWP did not differ too much near ground surface in different cases, while quite different near the bottom of the model ground. With the ground becoming denser in Case 2 and
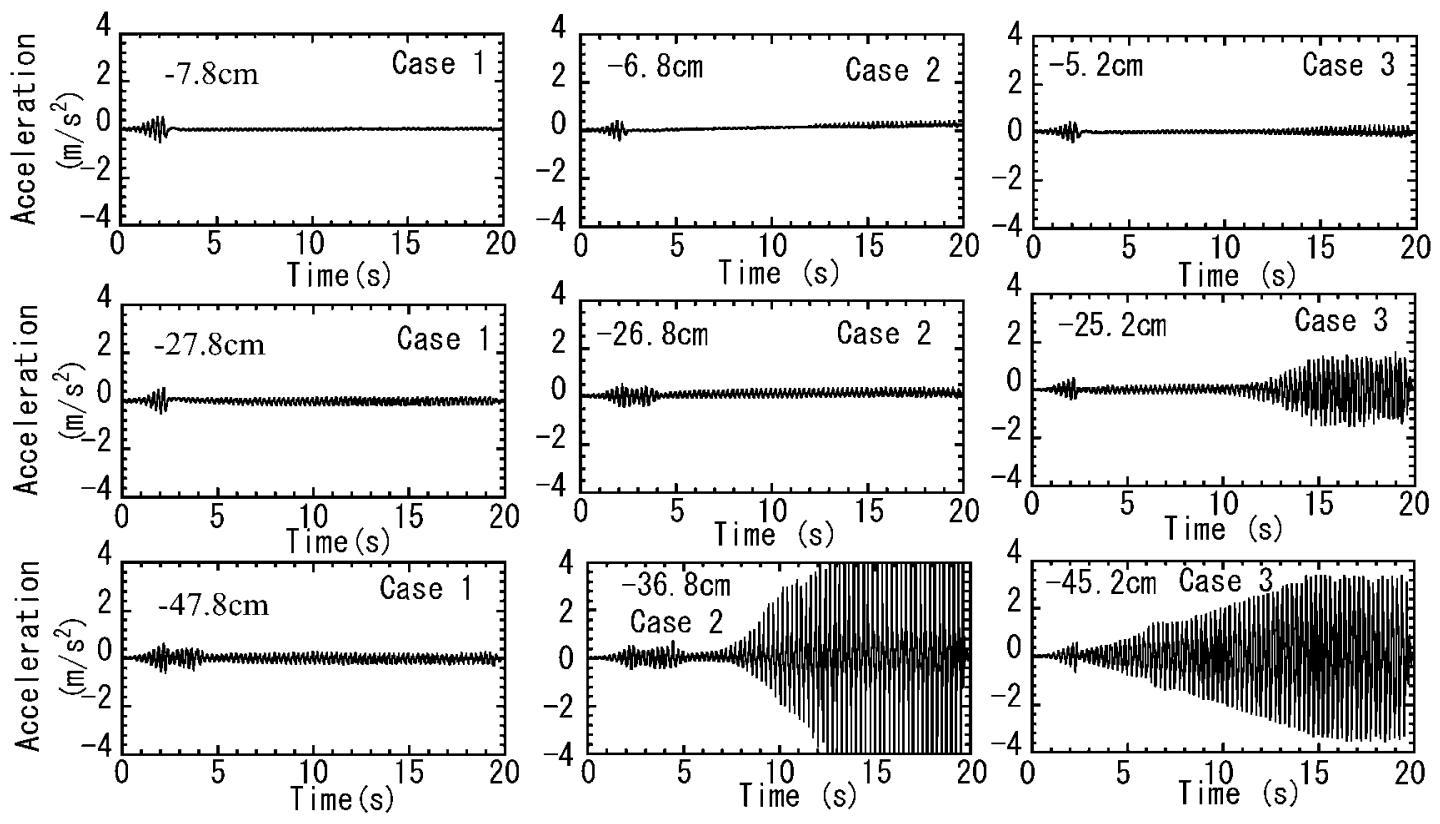

Fig. 7. Lateral acceleration of experimental results 

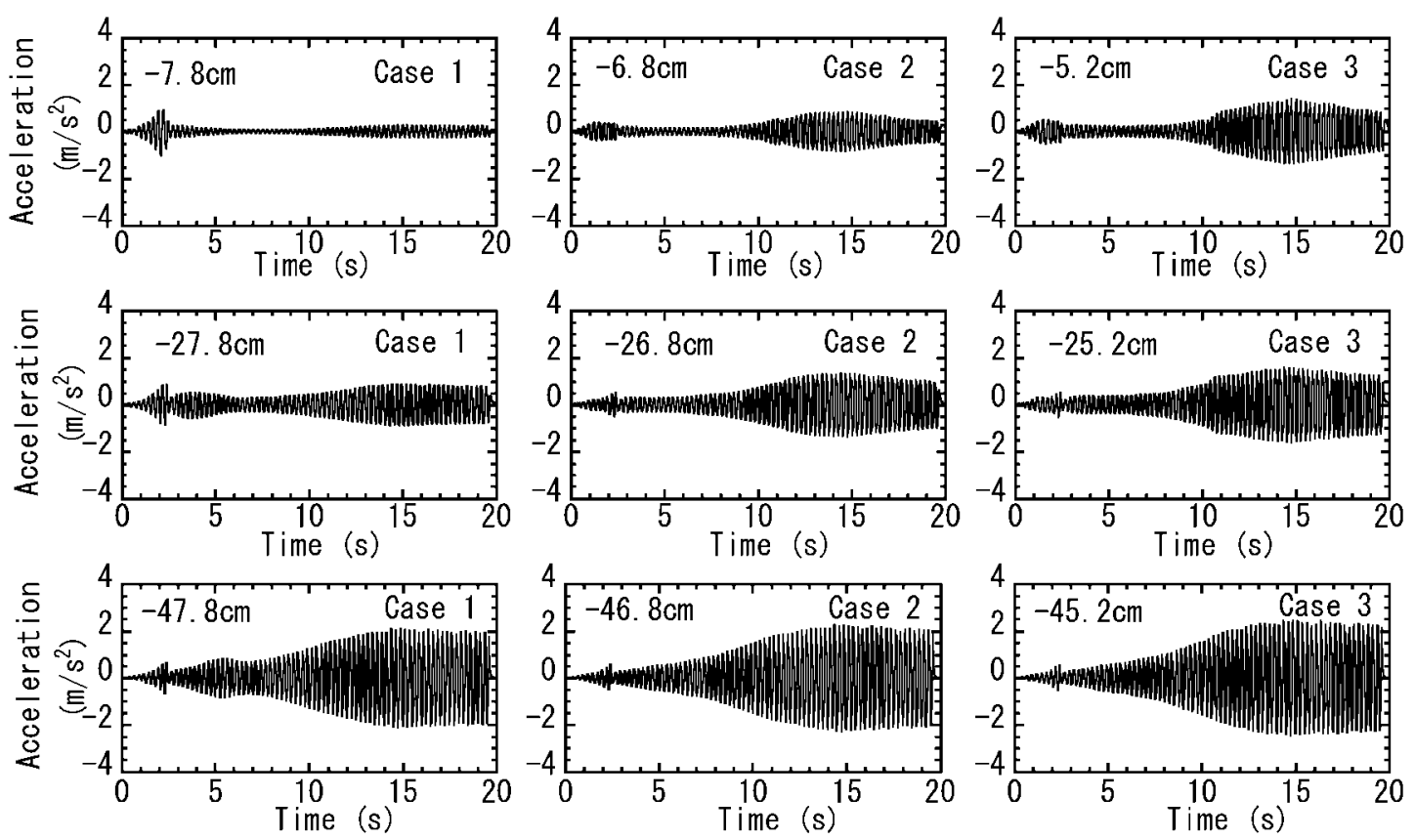

Fig. 8. Lateral acceleration of simulated results
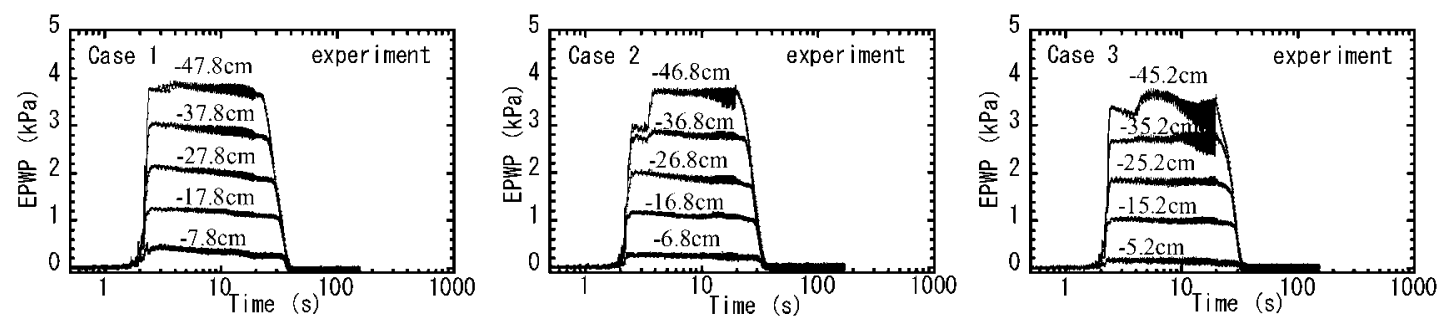

Fig. 9. Experimental results of EPWP
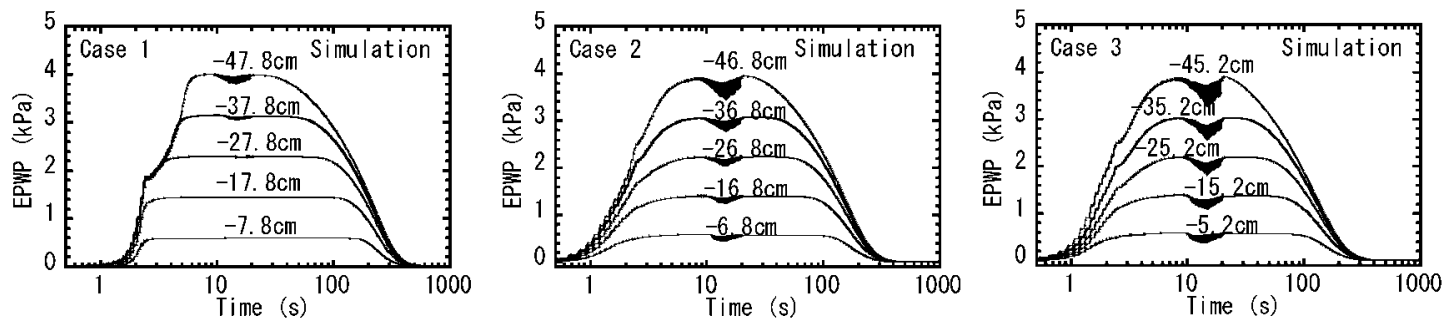

Fig. 10. Simulated results of EPWP

Case 3, the EPWP near bottom of the model ground began to vibrate. This is the typical characteristic which indicates that cyclic mobility is occurring. This phenomenon can be seen more clearly in Fig. 11, which shows the EPWP in different cases in one graph. It can also be seen from Fig. 11 that the dissipation times of EPWP in different cases were different. The denser the ground was the faster dissipation of EPWP.

Figure 10 shows the simulated result of EPWP at different depths in different cases. In Case 1, EPWP built up quickly to the highest value that is equal to the vertical effective stress. The value of EPWP kept almost constant until EPWP started to dissipate. In Case 2 and Case 3, however, when EPWP built up to a value close to vertical effective stress, the curves of EPWP began to vibrate and drop a little especially at deep places, which indicated that cyclic mobility began to occur in the sands of Case 2 and Case 3 due to the densification of the sand experienced the first liquefaction and consolidation process in Case 1. The above phenomena are in agreement with experimental results shown in Figs. 9 and 11. As to dissipation time for EPWP, however, simulated result was much longer than the corresponding experimental results. In experiment, the time necessary for the dissipation of EPWP was less than 30 seconds, while in the simulation, the dissipation time, which was calculated by a static 

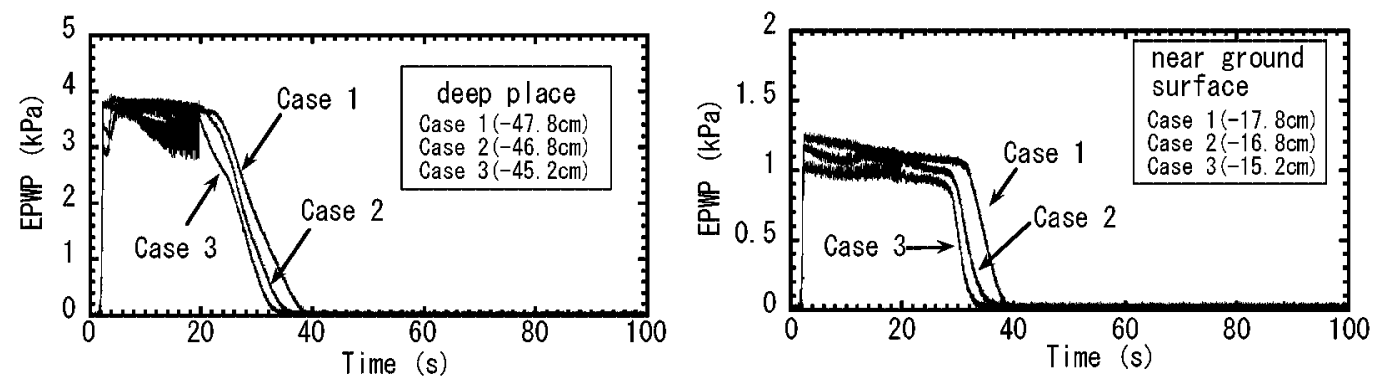

Fig. 11. Comparison of experimental EPWP in different cases
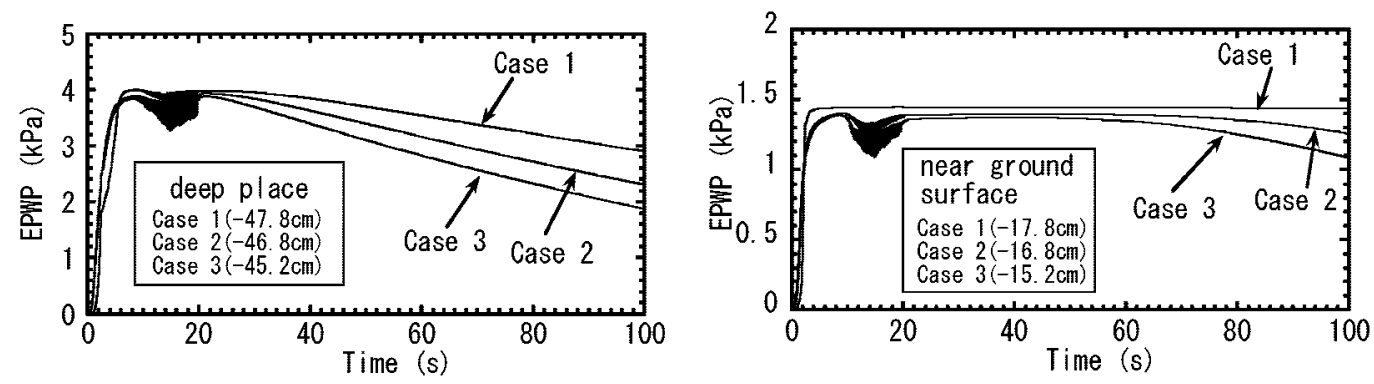

Fig. 12. Comparison of simulated EPWP in different cases

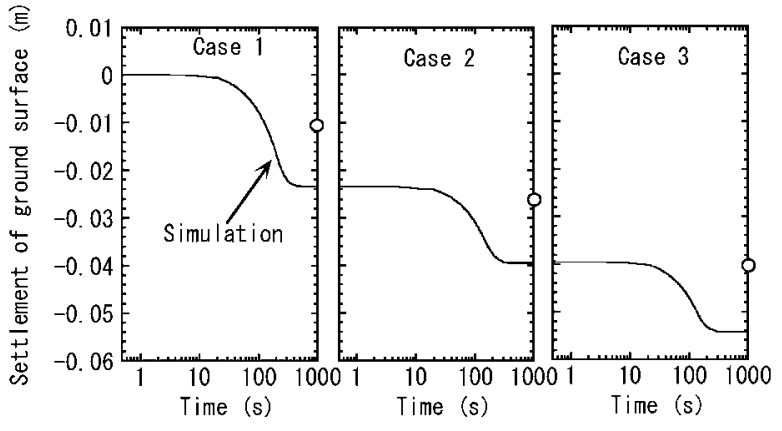

Fig. 13. Settlement of ground surface

consolidation analysis, lasted more than 300 seconds. This disagreement of dissipation time will be discussed in the next subsection. In spite of the disagreement of dissipation time, it can be seen from the simulated results shown in Fig. 12 that dissipation time depends on the density of sand, that is, the denser the sand is, the faster the dissipation of EPWP will be. This simulated result is in agreement with the experimental one shown in Fig. 11.

Figure 13 shows the calculated settlement of the ground surface. The small circles represent the measured settlements at the end of every case. The calculated settlement was larger than the measured settlement in Case 1, but agreed with the measured settlements in Cases 2 and 3 . This disagreement is probably due to the inaccurate evaluation of the stress condition for loose sand at very low confining pressure that can not be easily confirmed with conventional triaxial compression tests. Unevenness of material properties of model ground is also thought to be a reason for a non-uniform settlement in shaking table test, which may result in some errors in the measurements. The measured settlements mentioned here are the average values. On the other hand, the ground is assumed as uniform in the same depth.

In order to investigate mechanical behaviors of soil elements, three elements, element $6,16,26$ shown in Fig. 5 are picked up to see their stress paths and stressstrain relations. Figure 14 displays the stress paths of three elements in different cases. In Case 1, stress paths went directly towards zero effective stress state and there was no distinct cyclic mobility because the sand is loose. In Case 2 and Case 3, however, the distinct behavior of cyclic mobility did occur when stress path approached to zero effective stress. It also can be seen that the denser the sand is, the larger the shear resistance and the size of cyclic mobility loop will be. Figure 15 shows relations of shear strain and shear stress in different cases. In the first few loading cycles, ground did not liquefy and the shear strain of soil was small. After ground liquefied, the strain-stress curves in Case 1 became very flat with large shear strain and small shear stress, indicating that the stiffness of liquefied ground was very small in Case 1. In Case 2 and Case 3, however, the post-liquefaction stress-strain curves became steeper and steeper, which means that post-liquefaction stiffness of ground increased along with increase of the density of ground.

The above results have shown that numerical simulation by using the newly proposed model can describe different mechanical behaviors of sands that have experienced different liquefaction history and therefore has different densities. As mentioned before, the process in which sands become denser, is not simulated by changing the values of material parameters, but by the variation of the state parameters in the calculation, which is not assigned artificially but by strict calculation. The 

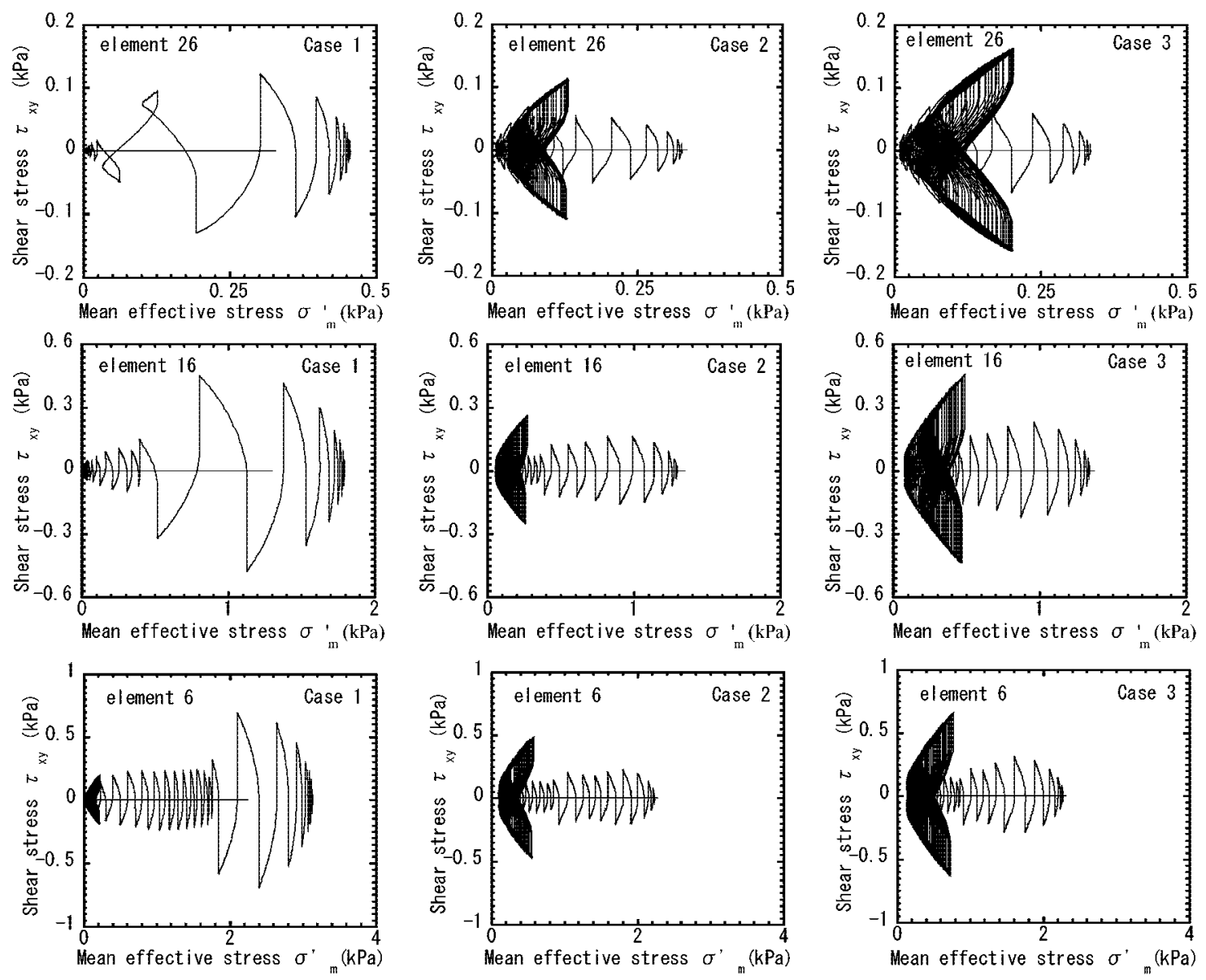

Fig. 14. Computed stress paths

state parameters $R^{*}$ and $R$, which evaluate the degree of structure and the degree of overconsolidation respectively, play very important role in determining the liquefaction behavior of the sandy ground. Figures 16 and 17 display the changes of the state parameters $R^{*}$ and $R$ in three elements. It can be seen that the value of $R^{*}$ increased quickly after shaking starts, and kept unchanged after reaching 1.0, indicating the complete collapse of soil structure. It is natural that structure of sands will never recover once it has collapsed. During shaking and liquefaction process, the state parameter $R$ that evaluates the degree of overconsolidation, decreased to a very small value due to the decrease in effective stress of sands. After ground motion was over, $R$ recovered with the increasing of effective stress due to the dissipation of EPWP in the process of consolidation. But $R$ can not recover to the initial value before shaking, which means that the sands now, are in a denser or higher degree of overconsolidated state than the initial state. In a word, the ground will lose its structure and become much denser or higher degree of overconsolidated state will occur after dissipation of EPWP. The different mechanical behaviors of sands with different densities subjected to repeated shakings are only dependent on the state of the sand, not on the material parameters. Figure 18 shows the changing history of stress-induced anisotropy, from which it is known that the stress- induced anisotropy of the sandy ground experienced liquefaction may accumulate to a certain value during the consolidation process but will change dramatically during the cyclic mobility loop at the next shaking process. The calculated void ratios are presented in Fig. 19, in which the changing process of the density of the sandy ground can be clearly pursued in the whole process of repeated shakings and consolidation.

\section{Some Discussions on the Dissipation Time of EPWP}

It is known that the dissipation time of EPWP after liquefaction is mainly dependent on the depth of liquefaction layer, the permeability of soil, and the stiffness of the soil. The first factor is very obviously and the other factors should be considered in detail. As mentioned in the previous subsection, the dissipation time of EPWP in present simulation is much longer than the experimental results. Two reasons can be considered for this disparity.

One is the permeability of sands. In the analysis, as the $20 \%$ particle diameter $\left(D_{20}\right)$ is 0.19 , according to the Creager's method, the permeability is assigned as $1.0^{-4}$ $\mathrm{m} / \mathrm{s}$, a commonly used value for Toyoura Sand. It is assumed in the analysis that ground keeps uniform throughout the whole process of repeated shaking and consolidation. But according to the observation of ground surface during experiment, some cracks (drainage 

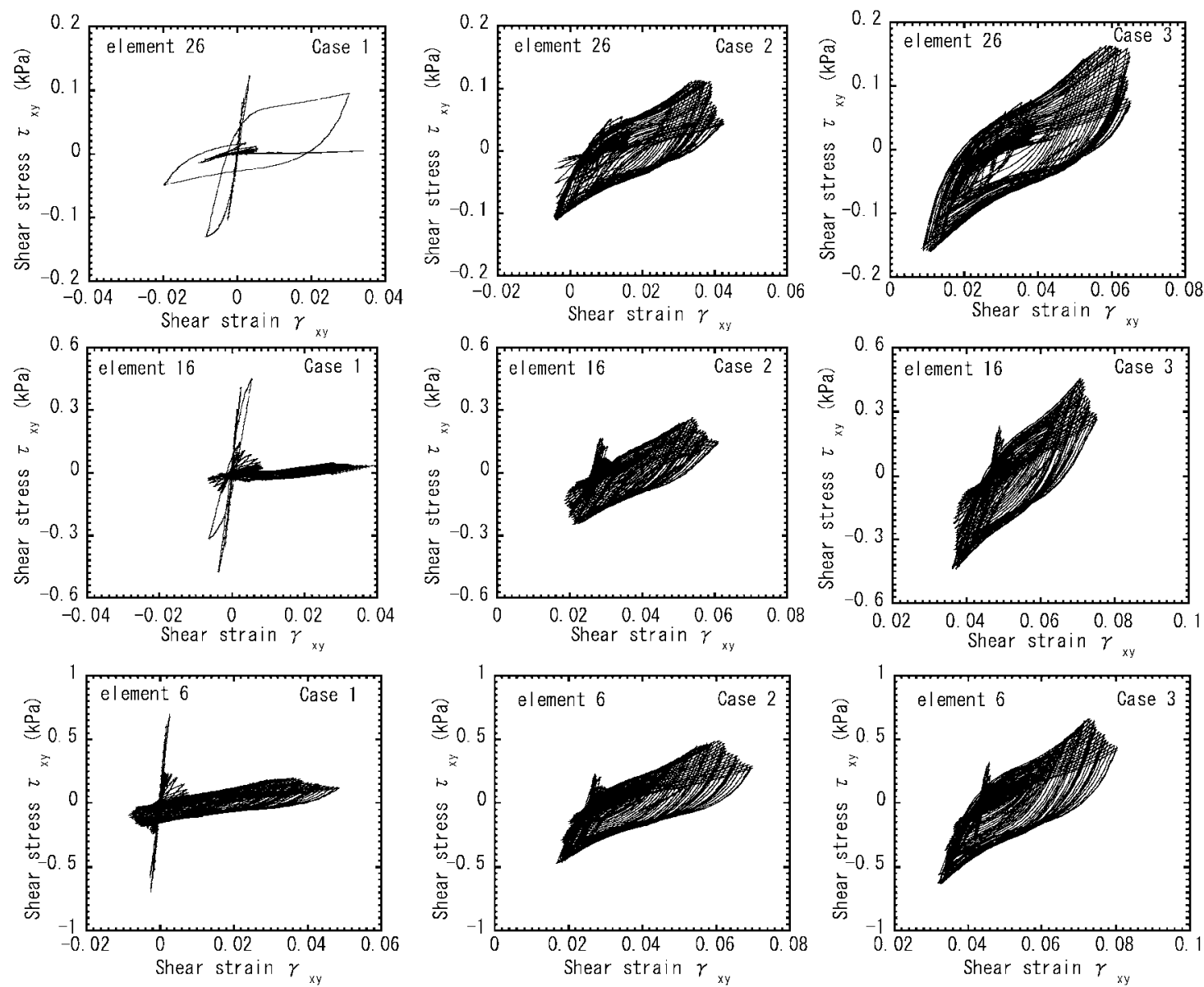

Fig. 15. Computed stress-strain relations

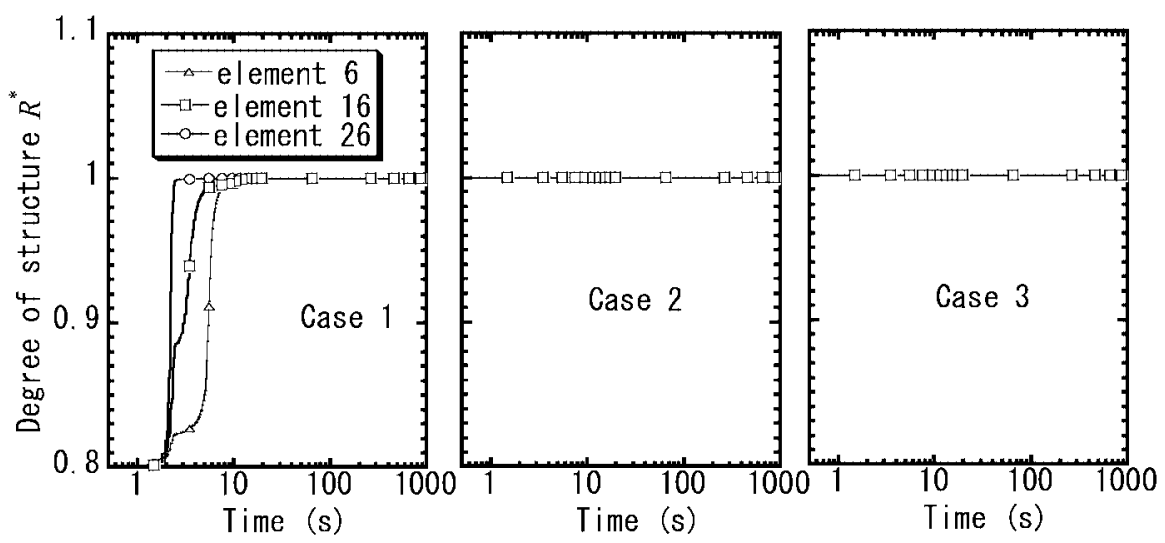

Fig. 16. Collapse history of structure $R^{*}$

piping) were observed in the ground surface, especially around the positions where measuring sensors were set and the side boundaries of shear box. Therefore, the ground will not be uniform after shaking and EPWP may dissipate more quickly from these cracks. In some places, even the phenomenon of sand boiling was observed. There are also some other experimental evidences of the increase of permeability during liquefaction, which can be found in literatures (Ishihara, 1993; Coelho et al., 2005). In order to investigate the influences of these cracks in the ground, we conducted a numerical analysis with the same sand but with a different finite element mesh, in which the existence of some channels for porewater dissipation is considered. These channels exist in the positions where the measuring sensors were set and the side boundaries of shear box, as shown in Fig. 20. The material of these channels are the same as the other sands except that the permeability is set as $1.0^{-3} \mathrm{~m} / \mathrm{s}$, one order larger than the usual sand. Figure 21 shows the history of EPWP calculated with the new FEM mesh. By comparing Fig. 21 with Fig. 11, it is clear that the channels for pore-water dissipation can greatly shorten the dissipation 

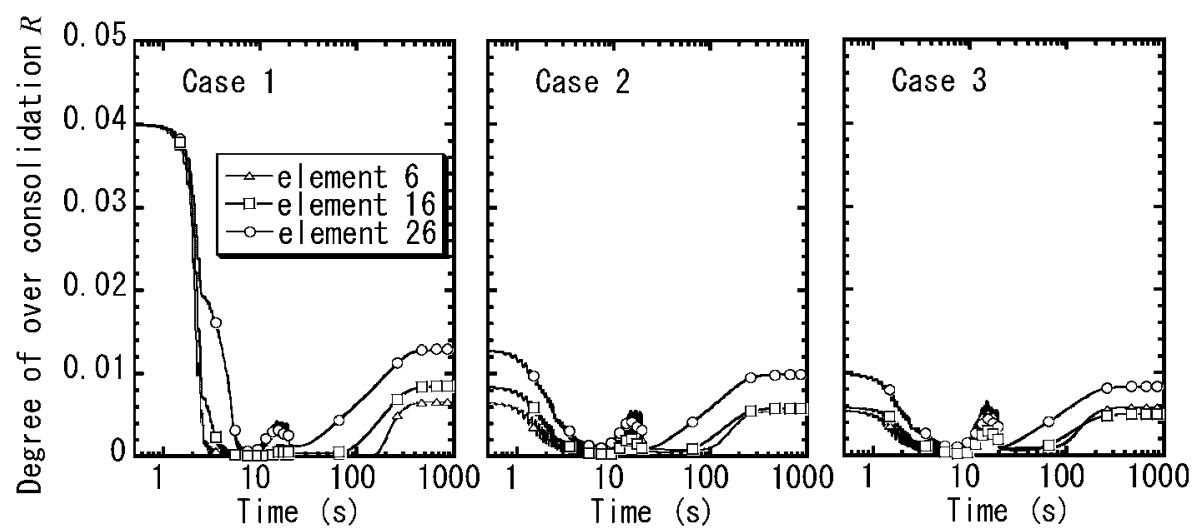

Fig. 17. Changing history of overconsolidation $R$

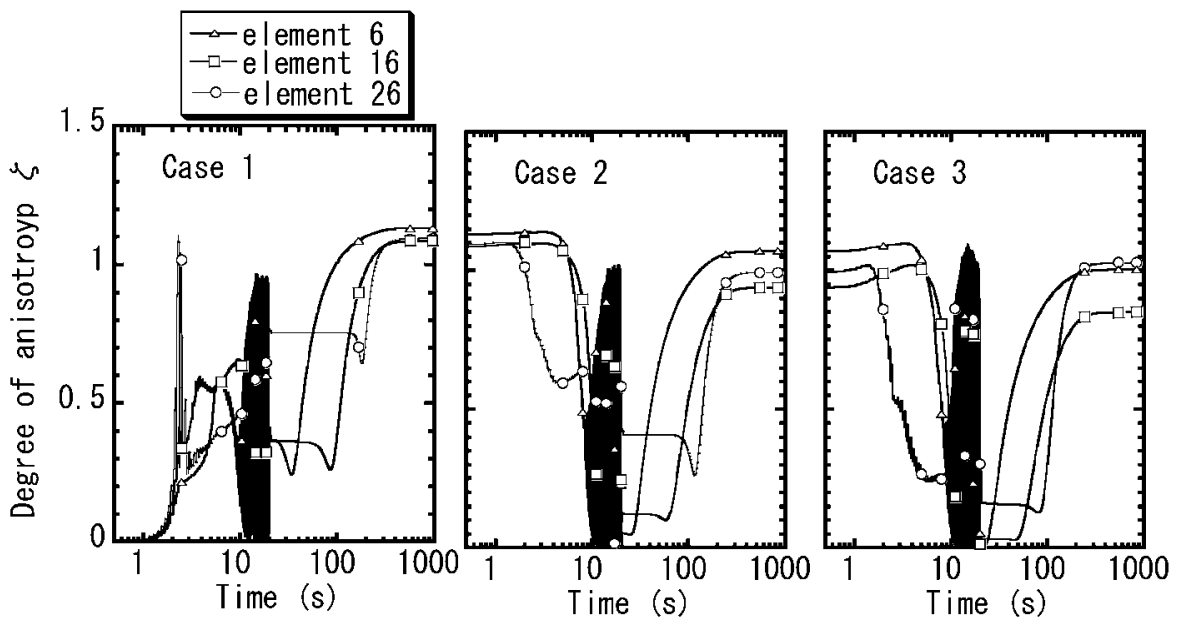

Fig. 18. Changing history of stress-induced anisotropy $\zeta$
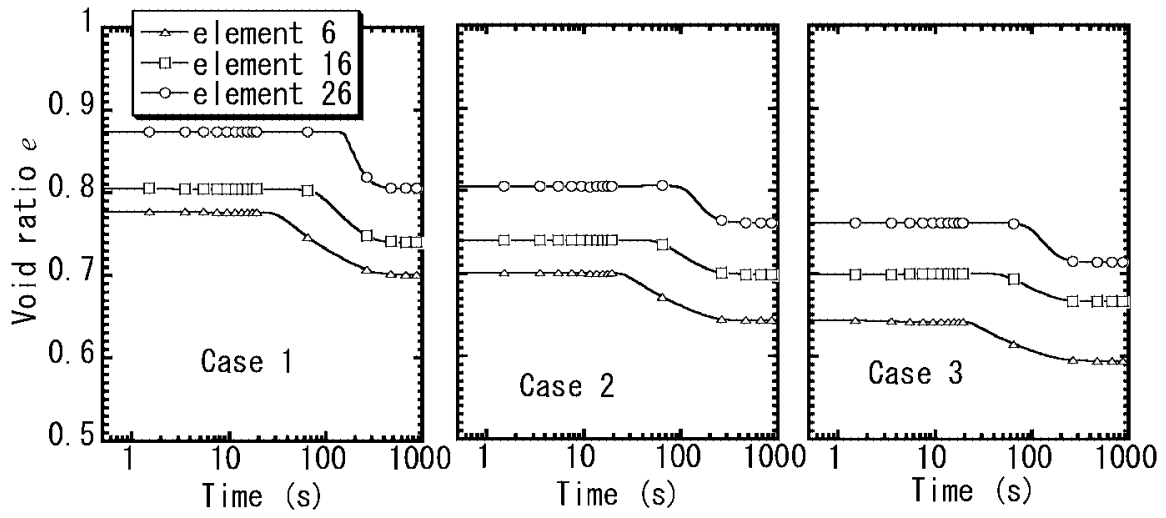

Fig. 19. Changing history of void ratio $e$

time of EPWP.

Another reason is considered to be the smaller value of swelling index $\kappa$. The value of $\kappa$ listed in Table 1 is determined by conventional triaxial tests on Toyoura Sand. But it is known that stiffness of soil is strain-dependent. Stiffness of soil at small-strain level is greatly larger than those measured in a conventional triaxial compression test. For instance, the value of swelling in$\operatorname{dex} \kappa$ is found to be 0.00023 in the shear-wave velocity test conducted in the work by Ye et al. (2006), almost four times as the value of those measured in conventional triaxial compression test. In order to investigate the influence of the stiffness on the rate of the dissipation of EPWP, the swelling index $\kappa$ is set as 0.00023 in each consolidation process while in the other process, it remains unchanged. Figure 22 shows the simulated results of EPWP. It is clear that the dissipation time was shortened by using the smaller value of $\kappa$. On the other 
hand, the difference in the rates of dissipation of EPWP at the grounds with different densities can not be described at all. The reason is that the increase of the stiffness of ground due to the densification after consolidation is much smaller than the increase by using a small value of $\kappa$ (27.8 times larger than original one) so that the influence of the densification can be omitted.

From the above two simulations, it is understood that the cracks within a liquefied ground and the evaluation of swelling index $\kappa$ at very small effective confining stress play a very important rule in estimation of the dissipation time of EPWP in the process of post-liquefied consolidation. It should be pointed out that some other factors may also affect the evaluation of the dissipation time of EPWP after liquefaction. For instance, unevenness of the physical properties of layered ground in shear box also may cause a non one-dimensional deformation of ground and flow of excessive pore water, which definitely

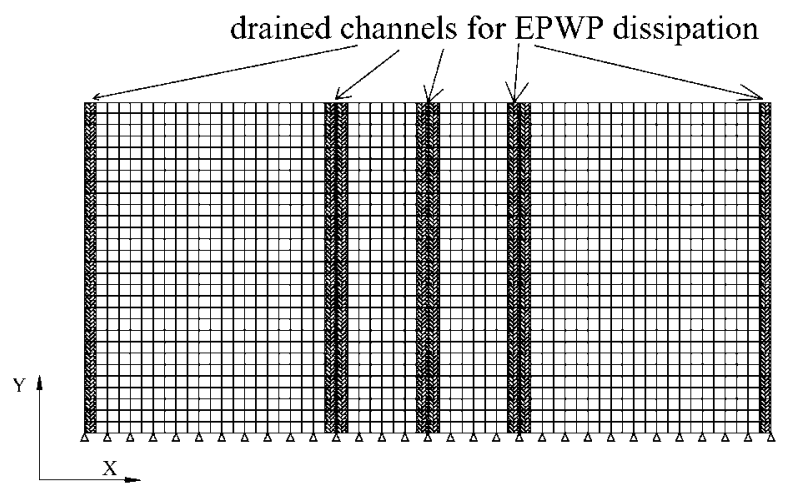

Fig. 20. New FEM mesh considering the existence of drained channels for EPWP dissipation affects the dissipation time.

\section{CONCLUSIONS}

Based on a newly proposed model, a repeated liquefaction-consolidation analysis was conducted to simulate a repeated shaking-table test on saturated sandy ground, considering the change of densities. Some conclusions are drawn as follows:

1. A repeated shaking-table test was conducted in Gifu University (Ye et al., 2006) to investigate the different mechanical behaviors of liquefied grounds with different densities. An original loose sandy ground was shaken three times in succession. Acceleration and EPWP in different places of the model ground were measured. It is found that once liquefied loose sandy ground will get denser in its consolidation process but is still possible to liquefy again in the next strong motion. It is also found that a ground is getting denser and denser in the repeated liquefaction-consolidation process, acceleration response of the ground will increase and the dissipation time of EPWP after liquefaction is getting shorter and shorter.

2. By comparing the numerical results with the experimental results, it is known that the analysis conducted in this paper is capable of reproducing uniquely the different responses of liquefied grounds with different densities during repeated shaking and consolidation. But the simulated dissipation time of EPWP is much longer than the experimental result.

3. In the first shaking, the effective stress paths of soil elements in loose sandy ground show no distinct cyclic mobility in the liquefaction process. When the
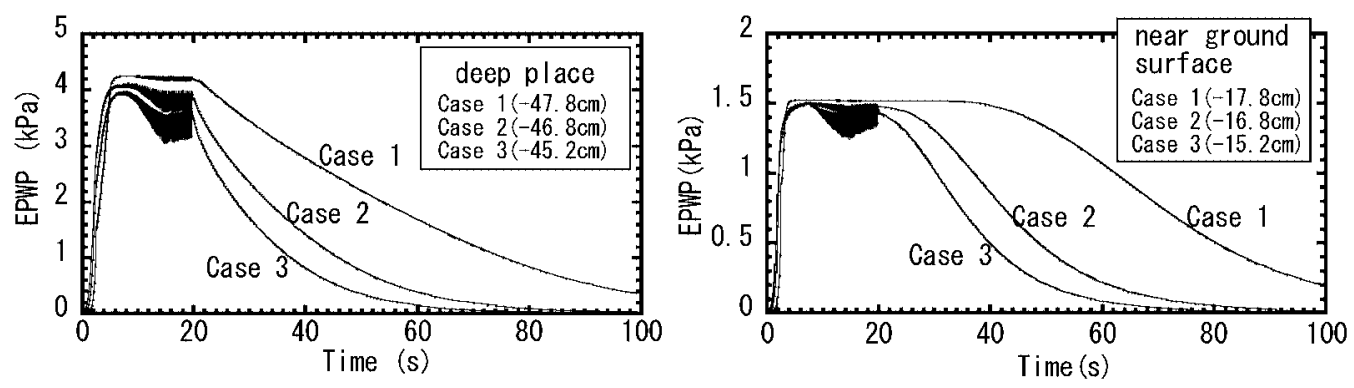

Fig. 21. Simulated results of EPWP considering the existence of drained channels for EPWP dissipation
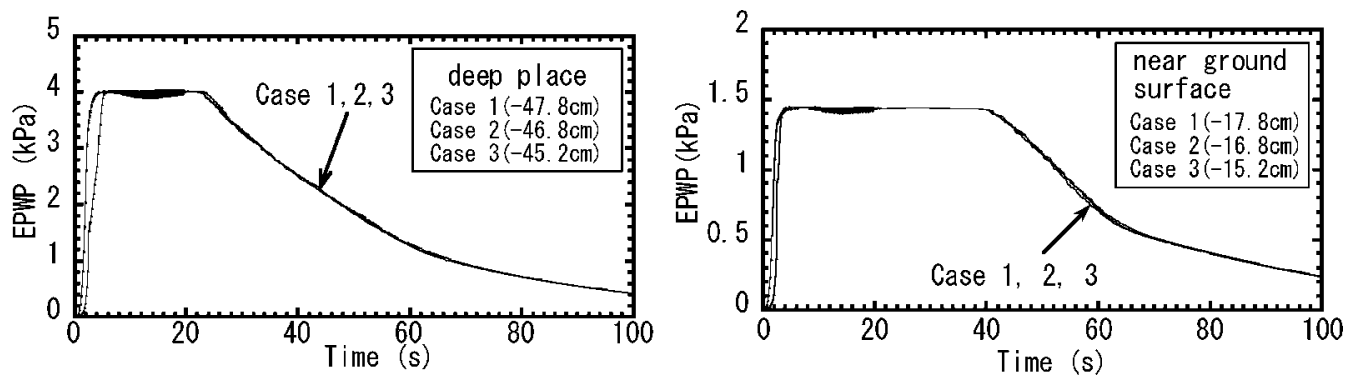

Fig. 22. Simulated results of EPWP with smaller value of $\kappa(\kappa=0.00023)$ during consolidation 
model ground is subjected to the second and third shakings, however, cyclic mobility does occur. It also can be found from stress paths that the denser the sand is, the larger the shear resistance and the size of cyclic mobility loop will be. The stress-strain relation curves become steeper when the ground becomes denser, implying that stiffness of ground increases with density of ground.

4. Three state parameters, $R^{*}, R$ and $\zeta$ which represent the degrees of structure, overconsolidation and stress-induced anisotropy respectively, play a very important role in determining the liquefaction and consolidation behaviors of the sandy ground. The ground will lose its structure and become much denser or have a higher degree of overconsolidated state after dissipation of EPWP. The different mechanical behaviors of sands with different densities subjected to repeated shaking and consolidation are only dependent on the state of the sand, not on the values of material parameters.

5. In simulating the whole process of repeated liquefaction and consolidation of sandy ground, though the density of the sand changes constantly, all values of material parameters are the same, which makes the numerical simulation being meaningful.

6. It is understood through numerical simulations that the cracks within a liquefied ground and the evaluation of swelling index $\kappa$ at very small effective confining stress play a very important role in estimating the dissipation time of EPWP in the process of post-liquefied consolidation.

\section{ACKNOWLEDGEMENTS}

The authors wish to express their thanks to $\mathrm{Mr}$. Yokawa, H., postgraduate student of Gifu University, Mr. Kondo, T., former students of Gifu University, and Mr. Yamada, H., former students of Nagoya Institute of Technology, for their efforts in conducting the shaking table tests discussed in this paper.

\section{REFERENCES}

1) Asaoka, A., Nakano, M. and Noda, T. (1998): Super loading yield surface concept for the saturated structured soils, Proc. 4th Eur. Conf. Num. Meth. Geotech. Engrg.-NUMGE98, 232-242.

2) Asaoka, A., Nakano, M. and Noda, T. (2000a): Superloading yield surface concept for highly structured soil behavior, Soils and Foundations, 40(2), 99-110.

3) Asaoka, A., Nakano, M., Noda, T. and Kaneda, K. (2000b): Delayed compression/consolidation of naturally clay due to degradation of soil structure, Soils and Foundations, 40(3), 75-85.

4) Asaoka, A., Noda, T., Yamada, E., Kaneda, K. and Nakano, M. (2002): An elasto-plastic description of two distinct volume change mechanisms of soils, Soils and Foundations, 42(5), 47-57.

5) Coelho, P., Haigh, S. and Madabhushi, S. (2005): Development, effects and mitigation of earthquake-induced liquefaction: A comprehensive study based on dynamic centrifuge modeling, Proc. 16th ICSMGE, 4, 2635-2639.

6) Elgamal, A., Yang, Z., Parra, E. and Ragheb, A. (2003): Modeling of cyclic mobility in saturated cohesionless soils, Int. J. Plasticity, 19, 883-905.

7) Hashiguchi, K. and Ueno, M. (1977): Elastoplastic constitutive laws of granular material, Constitutive Equations of Soils, Proc. 9th ICSMFE, Spec. Ses. 9, (eds. by Murayama, S. and Schofield, A. N.), Tokyo, JSSMFE, 73-82.

8) Hashiguchi, K. (1978): Plastic constitutive equations of granular materials, Proc. US-Japan Seminar on Continuum Mechanics and Statistical Approaches in the Mechanics of Granular-Materials, (eds. by Cowin, S. C. and Satake, M.), Sendai, JSSMFE, 321-329.

9) Hashiguchi, K. (1989): Subloading surface model in unconventional plasticity, Int. J. Solids and Structures, 25, 917-945.

10) Ishihara, K. and Kabilamany, K. (1990): Stress dilatancy and hardening laws for rigid granular model of sand, Soil Dynamics and Earthquake Engineering, 9(2), 66-77.

11) Ishihara, K. (1993): Review of the predictions for Model 1 in the VELACS program, Intern. Conf. Verification of Numerical Procedures for the Analysis of Soil Liquefaction Problems, (eds. by Arulanandan \& Scott), Balkema, Rotterdam.

12) Nakai, K. (2005): An elasto-plastic constitutive modeling of soils based on the evolution laws describing collapse of soil skeleton structure, loss of overconsolidation and development of anisotropy, Doctoral Thesis, Nagoya University (in Japanese).

13) Nishi, K. and Kanatani, M. (1990): Constitutive relations for sand under cyclic loading based on elasto-plasticity theory, Soils and Foundations, 30(2), 43-59.

14) Oka, F., Yashima, A., Shibata, T. and Kato, M. (1991): A finite element analysis of liquefaction of seabed due to wave action, GEO-COAST'91, 621-626.

15) Oka, F. (1992): A cyclic elasto-viscoplastic constitutive model for clay based on the non-linear hardening rule, Proc. 4th Int. Symp. Num. Models in Geomech., Swansea, 105-114.

16) Oka, F., Yashima, A., Kato, M. and Sekiguchi, K. (1992): A constitutive model for sand based on the non-linear kinematic hardening rule and its application, Proc. 10th World Conf. Earthquake Engineering, Madrid, Balkema, 5, 2529-2534.

17) Oka, F., Yashima, A., Shibata, T., Kato M. and Uzuoka, R. (1994): FEM-FDM coupled liquefaction analysis of a porous soil using an elasto-plastic model, Applied Scientific Research, 52, 209-245.

18) Oka, F., Yashima, A., Tateishi, A., Taguchi, Y. and Yamashita, S. (1999): A cyclic elastoplastic constitutive model for sand considering a plastic-strain dependence of the shear modulus, Geotechnique, 49(5), 661-680.

19) Pastor, M. and Zienkiewicz, O. C. (1986): A generalized plasticity, hierarchical model for sand under monotonic and cyclic loading, Proc. 2nd Int. Conf. Num. Models in Geomech., 131-150 .

20) Sekiguchi, H. (1977): Rheological characteristics of clays, Proc. 9th ICSMFE, Tokyo, 1, 289-292.

21) Yashima, A., Oka, F., Shibata, T. and Uzuoka, R. (1991): Liquefaction analysis by LIQCA, Proc. JGS Conf. Liquefaction of Ground and its Counter Measure, 165-174 (in Japanese).

22) Ye, B., Yokawa, H., Kondo, T., Yashima, A., Zhang, F. and Yamada, H. (2006): Investigation on stiffness recovery of liquefied sandy ground after liquefaction using shaking-table tests, soil and rock behavior and modeling, ASCE Geotechnical Special Publication No. 150, 482-489.

23) Zhang, F., Ye, B., Noda, T., Nakano, M. and Nakai, K. (2006): Explanation of cyclic mobility of soils: approach by stress-induced anisotropy, Soils and Foundations (accepted).

24) Zhang, J. M. and Wang, G. (2005): Fundamentals and constitutive model of large post-liquefaction deformation in saturated sand, Proc. 2nd China-Japan Geotechnical Symposium, (eds. by Huang et al.), Tongji University Press, 43-67. 